\title{
Controlled trial of synovectomy of knee and metacarpophalangeal joints in rheumatoid arthritis
}

\author{
ARTHRITIS AND RHEUMATISM COUNCIL AND BRITISH \\ ORTHOPAEDIC ASSOCIATION
}

\begin{abstract}
Arthritis and Rheumatism Council and British Orthopaedic Association (1976). Annals of the Rheumatic Diseases, 35, 437-442. Controlled trial of synovectomy of knee and metacarpophalangeal joints in rheumatoid arthritis. In a multicentre study patients with rheumatoid arthritis judged by prevailing criteria to be suitable for synovectomy of the knee or metacarpophalangeal (MCP) joints were randomly allocated to one of two groups. One group had the operation, the other was observed without operation from a notional corresponding date. 3 years later the outcome of synovectomy was compared with that of observation without synovectomy. Synovectomy of the knee was followed by significantly less pain and tenderness, smaller effusions, and smaller and less frequent erosions and geodes. By contrast, MCP joints were no better clinically or radiographically than those treated conservatively. The results have been compared with those of two other controlled trials, one concerned with the knee and MCP joints, the other only with MCP joints. In the present trial results were more favourable in the knee but comparable in the MCP joints with those reported in the first of these two trials but less favourable in the MCP joints than those observed in the second.
\end{abstract}

Synovectomy was discussed for the treatment of both rheumatoid arthritis and tuberculosis at a meeting of the Society of Surgery of Paris in 1900 (Mignon, 1900) and was sporadically used thereafter. The early 1960 s brought a resurgence of interest in the operation. For a time it was widely held that synovectomy was not only effective in the relief of symptoms, but was able to prevent or slow the evolution of irreversible damage to the structure of a joint. The validity of this belief could only be tested by controlled trials and at about the same time such trials were initiated in the USA by the Arthritis Foundation and in the UK by a joint committee of the Arthritis and Rheumatism Council and British Orthopaedic Association.

The preliminary designs of these two trials were compared at an international conference on synovectomy in Amsterdam in 1967 (Hijmans, Paul, and Herschel, 1969). As will be seen in the discussion, these two trials were sufficiently alike to allow a useful comparison of the results. The only other controlled trial, restricted to the metacarpophalangeal (MCP) joints, was conducted in Newcastle (Thompson, Douglas, and Davison, 1973) and again the data can be compared with those of the other two trials. The present trial compares the results of synovectomy with conservative methods in a weight-bearing joint, the knee, and a nonweightbearing joint, the MCP. Clinical results were measured by conventional means and progression of pathological changes by radiographs.

\section{Methods}

The criteria for entry into the trial are listed in Table $I$. Patients satisfying these criteria were chosen by a physician and subsequently examined by a surgeon. If the surgeon accepted that synovectomy would be appropriate, the next in sequence of a set of sealed envelopes held by each centre was opened and the allocation to synovectomy or control group made according to the instruction contained therein. Where possible synovectomy was performed within 2 months of allocation. A corresponding date 2 months after allocation was chosen as the base-line for assessment in the control group. No restrictions were placed on the drugs to be used during the trial in either group. Intraarticular injections were specifically allowed in the control group but no formal ruling about this form of treatment was ordained for synovectomy cases. Fortunately 
Table I UK synovectomy trial. Criteria for inclusion in trial

(1) Patient satisfies criteria of American Rheumatism Association for probable, definite, or classical rheumatoid arthritis

(2) At least one positive test for rheumatoid factor by any method within 3 months of entry to trial

(3) History of persistent swelling in the target joint for past 3 months at least

(4) Radiograph of target joint shows no more than minimal erosion or loss of joint space. In hand cases no MCP joint on the target side to show more advanced changes

(5) No contraindication to anaesthesia or surgery

(6) Involvement of other weight-bearing joints in knee cases not severe or extensive enough to hinder assessment of function in joint to be studied

(7) Where the target joint is the knee no more than $30^{\circ}$ loss of extension and no valgus or varus deformity present. (Many cases with loss of extension will fail to satisfy criterion (4)). Patients in whom patellectomy is indicated will also be excluded

injections were so rarely given in either group that any distortion of results by this measure can be ruled out. The regimen of postoperative management was left to the discretion of individual centres, though it was hoped that most would favour early mobilization.

After completion of the registration form the first general assessment was made on a date not more than 2 weeks before operation, or an equivalent date for controls; the second 2 months after operation in the synovectomy group; and the remainder annually from the date of operation or equivalent in controls. Radiographs were to be taken at the initial assessment and annually thereafter-anteroposterior of both hands in MCP cases, anteroposterior (resting and weight-bearing) and lateral of both knees in the knee group. In the event weightbearing films of the knees were often omitted. The method of scoring is given in the results.

Pain was scored on a 0, 1, 2, 3 scale and, in the synovectomy group, as better, worse, or unchanged as compared with its severity before operation. Range of motion was measured in degrees. Swelling in the MCP joints was recorded in the same way as pain. An attempt was made to record swelling of the knee by circumferential measurements at the upper margin of the patella and $7.5 \mathrm{~cm}$ proximal to this point. These measurements proved less informative than records of effusions scored on a $0,1,2,3$ scale. Tenderness was recorded on the same arbitrary scale, and grip with a standard pneumatic bag linked to a mercury sphygmomanometer and inflated to $20 \mathrm{mmHg}$ before the test. In the knee group any instability, lateral or anteroposterior, was noted. Synovectomy patients were asked whether they would have agreed to the operation had they known the outcome.

The erythrocyte sedimentation rate (Westergren) (ESR) and results of tests for rheumatoid factor (sheep cell agglutination test or latex test, or both) were also recorded, together with a list of drugs in current use. An additional special surgical assessment called for information of the state of the joint at operation. Unfortunately the number of cases in the trial was
Table II UK synovectomy trial

\begin{tabular}{llll}
\hline Joint & & Available for analysis & Withdrawn \\
\cline { 1 - 1 } Knees & & Synovectomy 22 & 1 \\
MCPs & Control 11 & 4 \\
& Synovectomy 13 (41 joints) & 0 \\
& Control 9 (28 joints) & 1 \\
\hline
\end{tabular}

Table III UK synovectomy trial. Arthritis and Rheumatism Council and British Orthopaedic Association. List of hospitals producing cases

Canadian Red Cross Memorial Hospital, Taplow

Leasowe Hospital, Liverpool

Middlesex Hospital, London

Northern General Hospital, Edinburgh

Nuffield Orthopaedic Hospital, Oxford

Rowley Bristow Orthopaedic Hospital, Pyrford

Royal National Hospital for Rheumatic Diseases, Bath

Royal Victoria Infirmary, Newcastle

St. Bartholomew's Hospital, London

St. Mary Abbots Hospital, London

Sheffield Centre for the Investigation of Rheumatic Dis- 옥 eases

Stoke Mandeville Hospital, Aylesbury

insufficient to allow these observations to be correlated $\stackrel{\mathbb{}}{\stackrel{(}{-}}$ with other data. The same obtains for information about $\overrightarrow{0}$ the immediate postoperative course, including complie ations.

\section{Results}

The number of cases available for clinical analysis is shown in Table II and the centres which contributed $\stackrel{\varrho}{\varrho}$ cases in Table III. Synovectomy (S) cases would $\stackrel{\square}{\unrhd}$ have matched controls $(C)$ in number if the original $\overrightarrow{\vec{D}}$ assumption that each centre would contribute $4 \stackrel{3}{3}$ cases had been realized. A group of 4 consecutive cases would have added 2 to each group. However, several centres contributed fewer than 4 cases (some considerably exceeded this number) hence the un- $\frac{0}{2}$ even numbers in the groups.

The data which follow were from patients who completed 3 years from operation, or equivalent $O$ date. In the analysis of the clinical data all differences between mean values were subjected to both음 Students' ' $t$ ' test and the Wilcoxon ranking test; in $>$ no case did the results differ. Patients withdrawn from the trial before completing 3 years and not included in the analysis numbered 6 , all in the kneegroup. One $\mathbf{S}$ case died and a second improved so $N$ much while awaiting operation (deferred for correction of anaemia) that the procedure was deemedo unnecessary. $\operatorname{In}^{\top} 2 \mathrm{C}$ cases it was" considered"unethicalo to withhold synovectomy, and 2 further $C$ cases failed to attend for assessment.

\section{KNEE}

On entry to the trial there were no significant differences between the groups in sex ratio, age $\bar{Q}$ 
distribution (mean 50.9 years in $S$ group, range $32-67 ; 50 \cdot 3$ years in $C$ group, range $36-61$ ), duration of arthritis $(5 \cdot 4$ years in $S$, range $0 \cdot 5-13 ; 9 \cdot 5$ years in $C$, range 1-41), ESR, or in any of the clinical measurements, though an excess of effusion in the $\mathbf{S}$ over the $\mathbf{C}$ group reached borderline significance $(P=0 \cdot 084)$. Serological data were not recorded for all patients but where titres in the sheep cell agglutination test were available, the $\mathrm{C}$ group started at a significantly higher level $(0.05>P>0.02)$. Data for the latex test were too scanty for analysis. There were no significant differences between the radiographs in the two groups at the first assessment.

\section{Pain}

It will be seen from Fig. 1 that there was a significant advantage to the $S$ cases with regard to pain at each yearly observation, with a trend towards a smaller difference at 3 years because of deterioration in the $\mathrm{S}$ and improvement in the $\mathrm{C}$ group.

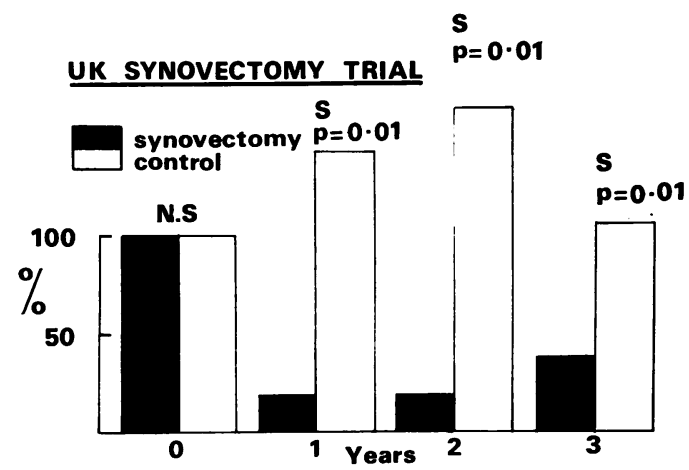

FIG. 1 Changes in pain in the knee expressed as percentage of score at entry in synovectomy and control cases on entry and at each yearly assessment. Base-line figures did not differ significantly and are expressed as $100 \%$

\section{UK SYNOVECTOMY TRIAL}

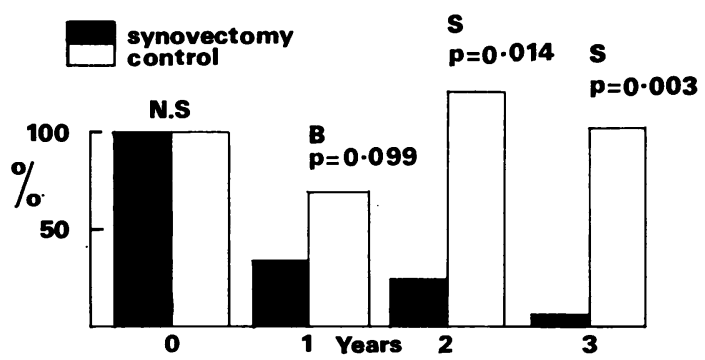

FIG. 2 Changes in tenderness in the knee expressed as percentage of score at entry in synovectomy and control cases on entry and at each yearly assessment. Base-line figures did not differ significantly and are expressed as $100 \%$

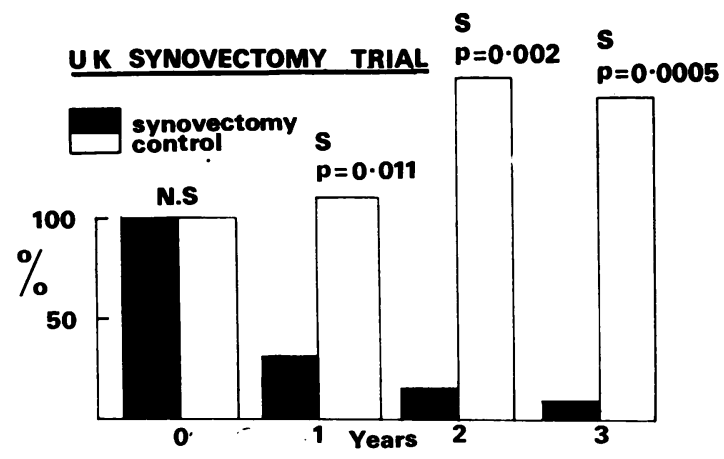

FIG. 3 Changes in effusion in the knee expressed as percentage of score at entry in synovectomy and control cases on entry and at each yearly assessment. Base-line figures did not differ significantly and are expressed as $100 \%$

\section{Tenderness}

The pattern for tenderness (Fig, 2) is similar to that for pain except that the excess of tenderness in the $C$ group at one year was only of borderline significance. However, the $S$ group did not show the same decline in its lead at 3 years as it did in the case of pain.

\section{Effusion}

The overall pattern (Fig. 3) is similar to that for tenderness with the $\mathbf{S}$ group maintaining its lead at each observation, though with a greater tendency for effusion in the $\mathrm{C}$ group to increase with time.

\section{Patient's opinion}

When asked if they would have agreed to the operation had they known the outcome, 3 said 'no' at one year, one was uncertain, 16 said 'yes'. At 3 years one said 'no' and 19 'yes'. Clearly no control data were available for comparison.

\section{Grip}

Measurements of grip were included as a crude index of general level of disease activity. There was a significant advantage to the $\mathrm{C}$ group at one year in the right hand but no difference at later assessments, or at any time in the left hand.

\section{Other clinical measurements}

Measurements which showed no significant differences between $\mathbf{S}$ and $\mathbf{C}$ were as follows.

(1) Range of movement-striking absence of change from mean base-line value in each group at each yearly assessment.

(2) Circumference at and proximal to patellachanges relatively small: final trend in favour of $\mathrm{C}$ group. 
(3) Lateral instability-little change, tended to increase in third year in $\mathbf{S}$ group.

(4) Anteroposterior instability-rarely present in either group: serial measurements favoured $S$ group.

(5) ESR - tendency to show a greater decrease at 3 years in $\mathrm{C}$ from a higher starting level and to remain unchanged in $\mathbf{S}$.

(6) Titre of rheumatoid factor-tended to fall in first year in $\mathbf{S}$.

(7) Involvement of knee in general exacerbationstoo few such exacerbations to determine degree of immunity, or otherwise, of joint under study.

\section{Radiographic changes}

Radiographs of 2 cases became available after analysis of clinical results had been completed and 33 sets of films $(22 \mathrm{~S}, 11 \mathrm{C})$ were read. Individual lesions were scored on a $0-3$ scale but as individual groups were small the final analysis was based simply on the presence or absence of deterioration in the sense of development of a lesion from the beginning or an increase in the severity of a lesion already present at entry to the trial. There were significant differences in favour of $S$ over $C$ in respect of erosions $(P=0.04)$ and cysts $(P=0.04)$. No significant differences emerged in respect of narrowing of joint space $(P=0 \cdot 57)$ or degenerative changes $(P=0 \cdot 19)$.

\section{MCP JOINTS}

As the number of patients was small the results for individual joints were pooled and analysed. This provided an $S$ total of 41 and a $C$ of 28 joints at the 3-year assessment. There was no real difference between the two groups on entry to the trial in respect of the factors already considered in the knee.

The results in these nonweight-bearing joints differed from those in the knees in that no significant differences emerged between the groups in any of the measurements. Thus $\mathbf{P}$ values for differences between the groups at 3 years were: pain $(0 \cdot 32)$, range of motion $(0 \cdot 13)$, swelling $(0 \cdot 395)$, tenderness $(0 \cdot 33)$, grip (right $0 \cdot 88$, left 0.87 ), ESR $(0.72)$, sheep cell agglutination test $(0 \cdot 22)$. There were, however, some significant differences at intermediate assessments: swelling, in favour of the $S$ group, at 2 years; range of motion, in favour of the $\mathrm{C}$ group at 1 and 2 years; tenderness, in favour of $\mathrm{C}$ group at 1 year.

The absence of any advantages to the $S$ group at 3 years, especially in terms of pain, might reasonably be expected to have been reflected in disappointment among patients. This was not so, in that all $13 \mathrm{~S}$ patients indicated at 3 years that they would have agreed to the operation had they known how they would fare. At 2 months they were unanimous, $\underset{\overparen{D}}{\overparen{D}}$ though at 1 and 2 years single patients (not the same $\exists$ individuals) said they would have declined.

\section{Radiographic changes}

Conforming with the clinical results no evidence emerged of any slowing of radiographic deterior-등 ation among joints treated by synovectomy when $\overline{\bar{c}}$ compared with control joints. The changes analysed, $\mathbb{\otimes}$ with the $P$ value for the differences between $S$ and $C$ groups in brackets, were: narrowing of joint space $(0 \cdot 57)$, erosion $(0 \cdot 30)$, subluxation or dislocation. $(0 \cdot 63)$, and cysts $(0 \cdot 99)$. Degenerative changes were $\overrightarrow{\vec{\omega}}$ too infrequent in both groups on entry and at $3 \stackrel{\omega}{\mathcal{N}}$ years to be analysed. Combining the two groups, erosion and subluxation or dislocation occurred or $\frac{0}{\omega}$ increased in about 1 case out of 3 , loss of joint space in 1 out of 2 , and cysts in 1 out of 6 .

\section{Discussion and conclusions}

The outcome of synovectomy has been reported by several workers but the results of the present trial $\bigcirc$ can most fruitfully be compared with the only two $\frac{0}{0}$ studies in which a control group was included. This $\mathbb{\Phi}$ restriction of discussion to controlled trials is not $\vec{\oplus}$ intended to imply that prolonged study of larg groups treated by synovectomy without contros. are incapable of yielding information of value. The present trial was designed to answer two questions? first whether or not synovectomy is superior to other forms of treatment in relieving symptoms, second whether or not it prevents or slows the evolution of the disease in a joint submitted to the operation.

Before discussing the results it is important to recognize the inherent limitations of the trial. First patients were contributed by 12 different centres. Inevitably the surgical technique must have varied. Treatment with drugs can be standardized in multicentre trials much more easily than a surgical procedure. Variations in technique would have been more readily discounted had individual centres contributed equal numbers of cases. Second, the 윽 synovectomy and control groups were numerically $\rightarrow$ unbalanced. Third, the total numbers observed were small; inter-group differences which did not reach $N$ statistical significance might have done so if more patients had been enrolled. Fourth, the trial was, of $\mathbb{N}$ necessity, not blind, with the important exception of $\mathrm{\omega}$ the analysis of radiographs. Fifth, synovectomy and $\bar{Z}$ control groups differed in ancillary treatment; in 0 addition to an operation the former had a period of $\Phi$ rest in bed and immobilization of the target joint $\stackrel{?}{?}$ supplemented by more intensive physiotherapy. 꿍 Sixth, the 'prophylactic' potential of synvectomy was assessed largely by radiographs. Surgeons with $\stackrel{?}{+}$ experience in rheumatoid arthritis often see quite $\stackrel{\mathbb{Q}}{\varrho}$ 
advanced irreversible damage on opening joints which had appeared intact radiographically. For practical purposes radiographs in a multicentre trial such as this had to be standard projections. Serial biopsies were not deemed to be justifiable and arthroscopy was not sufficiently widely available to be included in the design.

The results now to be discussed emerge from observations over a period of 3 years in patients satisfying certain criteria. Different criteria and a longer period of observation might yield different conclusions. The differing patterns of results in the knee and MCP joints vindicate the original decision to study two joints and invalidate any general statement bearing on the outcome of synovectomy irrespective of site.

After 3 years knees treated by early synovectomy were as a group less painful and tender than those treated conservatively and had less effusion. The results of synovectomy were more favourable than those found in a trial in the USA organized by the Arthritis Committee of Evaluation of Synovectomy (McEwen and O'Brian, 1974), in which only soft tissue swelling was significantly less 3 years after operation than in control cases. This trial in the USA was similar in design to that reported here in comparing synovectomized and conservatively treated knees in separate patients but incorporated an additional comparison between one synovectomized and one control knee in individual patients with symmetrical involvement. In the UK trial radiographic changes favoured synovectomy in that erosions and cysts were smaller or absent after 3 years than in control patients at the corresponding time. There was no evidence that knees treated by synovectomy tended to develop degenerative changes more often than control knees. The results appear to justify synovectomy of the knee in cases satisfying the criteria used in this trial. It certainly did not halt irreversible changes in all treated joints but significantly slowed radiographic deterioration (and presumably the evolution of the pathological process) in some. Furthermore, it often appears to provide a minimum of 3 years' important relief of symptoms without prejudicing future reconstructive surgery.

By contrast, results in MCP joints were discouraging. Joints treated by synovectomy showed no advantage over control joints at 3 years either clinically or radiographically. It is interesting and instructive that almost all patients affirmed in retrospect that they would have agreed to have the operation had they known the outcome. Among possible explanations for this, so much at variance with the other clinical assessments, two may be considered. First that synovectomy was beneficial but the methods for measuring its effects were too crude to establish this; second that patients felt that a procedure involving a period in hospital, considerable inconvenience, and a moderate amount of discomfort must have been helpful. The trial in the USA (McEwen and O'Brian, 1974) which included proximal interphalangeal as well as MCP joints likewise showed no clinical advantage from synovectomy at 3 years-the radiographs will be reviewed at 5 years. In an earlier controlled trial in the UK (Thompson and others, 1973) synovectomy of MCP joints produced more favourable results. At 2 years joints treated by synovectomy were significantly better than control joints in terms of patient's assessment, swelling, power and palmar pinch grip, and pain. This trial included a second control group. treated by splinting; again synovectomy proved clinically superior, except in reducing swelling. As in the present trial radiographs showed deterioration of equal extent in all groups. The more favourable clinical outcome in this trial is not explained by the shorter period of observation except possibly in the case of swelling, where at 2 years joints in the synovectomy group in the present trial were significantly less swollen. It may be relevant, however, that in this singlecentre trial (Thompson and others, 1973) the technique of synovectomy and assessment was presumably more uniform than in the present trial.

No better explanation for the discrepancy in clinical results between this trial and the USA and UK multicentre studies can be offered and no other conclusion reached than that the last two provide no support for synovectomy as a useful form of treatment for the MCP joints in rheumatoid arthritis. As in all therapeutic trials where comparisons are made between groups of patients pooled data may conceal individual patients for whom synovectomy was advantageous. The onus for seeking criteria by which such patients can be identified lies with those who continue to favour it.

The joint committee of the Arthritis and Rheumatism Council and British Orthopaedic Association which sponsored the trial first met in 1966 under the Chairmanship of the late Dr. W. S. C. Copeman, C.B.E. The original representatives from the ARC were Professor J. J. R. Duthie, Dr. Alan Hill, Dr. E. Lewis-Faning, and Dr. Michael Mason, and from the BOA Mr. Philip Bliss, Mr. Alford Dornan (later succeeded by Mr. F. W. Taylor), Professor Robert Duthie, Mr. H. B. S. Kemp, Sir Henry Osmond-Clarke, the late Mr. Geoffrey Platt, the late Mr. D. L. Savill, and Mr. D. R. Sweetnam.

The responsible consultants in the Units listed in Table III were Professor E. G. L. Bywaters, Dr. T. R. Littler, Dr. Mary Corbett, Professor J. J. R. Duthie, Dr. Alastair Mowat, Dr. A. B. Myles, Dr. Allan St. J. Dixon, Dr. Malcolm Thompson, Dr. H. Wykeham Balme, Dr. E. N. Coomes, Dr. G. R. Newns, and Dr. Alan Hill. The co-ordinator was Dr. Hill (assisted by 
Mrs. Ruth Kerr, research secretary supported by the ARC).

Analysis of clinical data was undertaken by Dr. E. Lewis-Faning. Radiographs were read by Mr. A. R Taylor, and the results analysed by Dr. A. Barr of the
Oxford Regional Health Authority. The organizing committee is grateful to the surgeons who performed the synovectomies, to the heads of departments and their medical and secretarial staff who collected the data, and to the patients who so willingly collaborated.

\section{References}

Hijmans, W., Paul, W. D., and Herschel, H. (1969) (eds.) 'Early Synovectomy in Rheumatoid Arthritis'. Excerpta Medica, Amsterdam

McEwan, C., AND O'Brian, W. B. (1974) J. Rheumatol., Suppl. 1, 107 (A multi-center evaluation of early synovectomy in the treatment of rheumatoid arthritis) (Abstr.)

Mignon, A. (1900) Bull. Soc. Chir. Paris, 26, 1113 (Synovectomie du genou)

Thompson, M., Douglas, G., AND Davison, E. P. (1973) Proc. roy. Soc. Med., 66, 197 (Evaluation of synovectomy in rheumatoid arthritis) 